\title{
An Efficient and Robust High Efficiency Video Coding Framework to Enhance Perceptual Quality of Real-Time Video Frames
}

\author{
Murthy SVN \\ Research Scholar \\ Department of Computer Science \& Engg. \\ Jain University, Bangalore, India
}

\author{
Dr. Sujatha B K \\ Professor \\ Department of Telecommunication \& Engg. \\ MS Ramaiah Institute of Technology, Bangalore, India
}

\begin{abstract}
Different level of compression on real-time video streaming has successfully reduced the storage space complexities and bandwidth constraints in the recent times. This paper aims to design and develop a novel concept towards the enhancement of perceptual quality of a real-time video frames. The proposed model has been experimented considering multilevel compression operation using $\mathbf{H . 2 6 5}$ where avi moving frames standards play a crucial role. The study also applies a novel concept of High-efficiency video coding (HEVC) for adaptive live video streaming over a mobile network. The proposed study aims to formulate a multi-level optimization for HEVC to enhance the performance of both encoding and decoding mechanisms at the client as well as server side to ensure higher compression rate. The experimental outcomes also show that the proposed protocol achieves better performance ratio and overall; throughput in comparison with conventional H.263, H.264 by enhancing the perceptual quality of .avi format realtime video frames.
\end{abstract}

\section{Keywords-H.265; HEVC; Video Coding; Compression}

\section{INTRODUCTION}

Video encoding is the way toward diminishing the measure of information required to interpret to an advanced video signal, preceding transmission or capacity. The corresponding operation, decompression or disentangling, recoups an advanced video signal from a compacted representation, before the presentation. Advanced video information tends to take up a lot of capacity or transmission limit thus video encoding and translating, or video coding is a key attribute for any application in which transmission data transfer capacity is compelled and constrained by a limited bandwidth. HighEfficiency Video Coding (HEVC) is right now the freshest video coding standard of the ITU-T Video Coding Experts Group (VCEG) and the ISO/IEC Moving Picture Experts Group (MPEG) [1][2]. The principle objective of the HEVC institutionalization exertion is to empower fundamentally enhanced compression metric execution in respect to existing models - in the scope of half piece rate decrease for equivalent perceptual video quality. HEVC guarantees to diminish the general expense of conveying and putting away video resources while keeping up or expanding the nature of the experience for the viewer. These are the two advantages of HEVC in the spilling space. The principal identifies with encoding existing SD and HD content with HEVC as opposed to H.264, empowering cost reserve funds and the capacity to stream higher quality video to lower bit rate associations. The second identifies with opening up new markets for ultrasuperior quality (UHD) recordings. This research study discusses the retention of higher quality of video file and traffic management when a video is compressed using HEVC over the wireless mobile network. The present pattern in video utilization obviously demonstrates that the officially expansive amount of video material conveyed over communication channels, advanced systems, and bundled media is going to increment in the coming years. As an impact of the developing notoriety, the client's interest for expanded determination and higher quality is driving the endeavors of the technological improvement. Starting here of perspective, the advancement of video obtaining and show advances is much quicker than that of system, the users' demand for increased resolution and higher quality is driving the efforts of the technological development. From this point of view, the evolution of video acquisition and display technologies is much faster than that of network capabilities. Users would like to watch streaming videos on mobile phones on the go. Though H.264 is succeeded in providing a good motion picture in Television, High Definition Television (HDTV), and Full High Definition Television and even to web based applications. But it requires higher bit rate and hence it is failed to deliver high definition videos to mobiles and to tablets.

Therefore, it is an open research problem to conceptualize, design and device a mechanism involves into H.264/AVC encoding processes to achieve higher performance with least computational overhead by low complex implementations. Typically, an H.264/AVC encoding process involves removal of spatial, temporal and statistical redundancy of video signal. The transformation of macro blocks, (a basic coding unit of 16 $\mathrm{X} 16$ block of displayed pixel) by quantization of transform domain (spatial frequency components/ co-efficient) from spatial domain provides a considerable amount of compression. The proposed study aims to design an efficient and novel video compression technique namely HEVC based optimized encoding tool to improve the perceptual quality of real-time encoded video streams on a mobile network. The paper is organized as follows Section II discusses the recent studies towards conventional real-time video compression mechanisms (i.e. using H.263, H.264, H.265) which are followed by problem description in Section III. Section IV discusses proposed system followed by a discussion of algorithm 
implementation in Section V. Section VI discusses the result analysis followed by conclusion in Section VII.

\section{LITERATURE SURVEY}

This section discusses the existing research work being carried out using H.265 video compression standard. Most recently, Chen et al. [3] have introduced a decoder by H.265 using a hardware-based architecture with supportability of the parallel processing. The outcome of the study evaluated on decoding speed shows that presented technique can support decoding of 8K UHD. Dias et al. [4] have presented a study by incorporating H.265 protocol for enhancing the quantization with the aid of rate distortion theory. The presented study has used spatial-based solution to enhance the visual perception of the signal. The study has been testified with mean opinion score and multimedia quality. The similar direction of work by adopting rate-distortion theory has been implemented by Nguyen and Marpe [5]. The authors have used the image as data to be compressed using H.265 protocol. The study outcome found that mean of bit rate saving using JPEG is quite high compared to other compression technique along with H.265. Panayides et al. [6] have presented a study recently that studied comparative analysis of various existing signaling protocols on H.265 taking the case study of medical video dataset. Trzcianowski [7] have also presented a study that has focused video coding for legacy protocol e.g. advances video coding.

A very interesting study was put forward by Ye et al. [8] have elaborated about the usage of UHD files and its management over the networks. The author discussed that usage of H.265 can save around $64 \%$ of the bit rate as compared to high definition and standard definition video. He et al. [9] have presented a new approximation technique for ensuing processing of UHD files with a major focus on minimizing computational complexity about search-techniques for optimal coefficients of resolutions. The technique was implemented on hardware-based approach over VLSI architecture. Ahn et al. [10] have discussed various optimization techniques using H.265 with the aid of parallel processing. Also, the authors have presented a task scheduling technique along with slicing process in parallelization over multicourse using H.265. Blasi et al. [11] have presented a technique for minimizing the complexity related to motion compensation. Diaset al. [12] have presented an FPGA-based study for performing compression using H.265 protocol using an integrated scheme of the efficient computational process along with 2D transforms. $\mathrm{Mu}$ et al. [13] have presented a cascaded technique for legacy protocols e.g. H.264/AVC and was subjected to H.265 encoding process to extract the encoding coefficients. The focus was laid on enhancing the speed of coding tree unit. Hence, there are various studies that have focused on using video compression algorithm. The techniques are associated with advantages as well as a limitation. However, it has acted as better guidelines for designing a novel protocol of video compression.

\section{PROBLEM DESCRIPTION}

Existing techniques don't emphasize on developing ondemand video compression techniques which ignore the computational complexity. Existing research work towards video compression doesn't have the explicit design of wireless communication channel and its associated problems. The entire compression algorithm designed till date seriously lacks multilevel optimization, which will mean that the existing algorithm can offer one round of better solution and cannot go beyond that. Although PSNR is found to be used in literature, its values are less emphasized on $8 \mathrm{~K}$ or UHD data quality.

\section{RESEARCH METHODOLOGY}

The proposed study aims to design and develop an integrated HEVC standard prototype to enhance the perceptual quality of transmitted video frames using H.265 video compression technique. The resulting compressed video frames also support $8 \mathrm{~K}$ (UHD) resolutions features, and it also maintains the PSNR ratio in between the range of 35-40 dB. The following figure 1 depicts the architecture of the proposed model and the overall flow towards ensuring the better retention of $8 \mathrm{~K}$ (UHD) videos after encoding. However, there are conventional techniques used to optimize the $8 \mathrm{~K}$ resolution efficiency, but the proposed H.265 prototype | Codec maximizes the perceptual quality and the resolution of an encoded video considering the optimal trade-off. The H.265 protocol runs over a distributed real-time TCP/IP communication scenario where $\mathrm{n}$ number of clients is connected to a server using TCP/IP protocol stack based communication terminals. Hence, an analytical methodology has been conceptualized and experimented by streaming standard definition (SD), High Definition (HD) and Ultra High Definition (UHD) in a real time scenario. The proposed study is precisely focused on designing a multi-level HEVC video compression technique to compress $8 \mathrm{~K}$ videos on real time transmission stage using H.265 without affecting its original quality. The proposed model performs both encoding and decoding in the server and receiver sides respectively, but the compression of the transmitted video happens during the transmission scenario configured by installing and setting up DASH servers for client and receiver units.

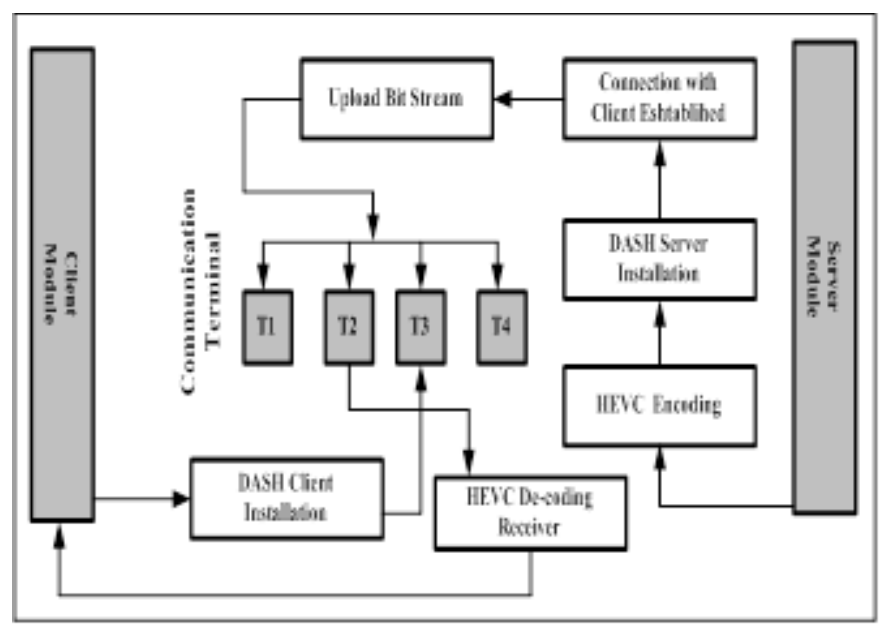

Fig. 1. Proposed Architecture

Apparently, the real-time streaming videos during the buffering time which are usually the time requires to decode the encoded or compressed $8 \mathrm{~K}$ videos are mostly get affected by the noisy environment of the wireless communication 
mediums, and it also degrades the quality of the video even to a minor extent. To address the above-stated issue of 8K (UHD) video frames transmission over a wireless channel DASH server and its functionality initiated in both client and the server side for video quality enhancement. The proposed system initiates a dynamic adaptive streaming over HTTP (DASH) server for transmitting the encoded 8K (UHD) video frames over a wireless channel. It has also been implemented over fast data communication scenario, which enables high quality streaming media contents from conventional HTTP servers using a constraint band-width capacity. The design principles associated with a DASH server configuration and real-time integration is illustrated below.

\section{A. Design Principle of DASH Servers}

The principles of DASH servers gained a significant market adoption for the real-time video streaming and compression protocols such as H.265. HTTP-based streaming should be closely inclined on HTTP server based progressive downloading scenario. HTTP based high-resolution video streaming processes are found to give a normative description of media presentation over wireless channels. The proposed HTTP based HEVC protocol incorporates streaming of encoded 8K (UHD) video frames on real-time and on-demand services. It also uses the concept of progressive download for on-demand media delivery from HTTP servers. The proposed system has been designed and experimented considering the following conditions which have been considered referring the conventional design flaws of HTTP/1.1 web server-based progressive downloading.

- The proposed system should effectively utilize the bandwidth even if a client unit terminates the data connection to streaming of the encoded $8 \mathrm{~K}$ (UHD) videos frames and switch over another content.

- The proposed HEVC protocol is modeled based on the adaptive bit-rate scenario and it also supports the live media services compatible with live video streaming over HTTP-dash addresses.

- It has also been evaluated over a wireless mobile communication medium which ensures reliability and the deployment simplicity of TCP/IP protocol stacks for efficient connection establishment in between client and server units.

The proposed system has also been integrated with The Content Distribution namely CDN Network to reducing the compression and downloading latency of media files. However, initially HTTP streaming has been used in 3GPP adaptive multimedia streaming, hence due to its optimal bandwidth utilization efficiency and adaptive bit-rate switching it is further integrated with 3GPP TS 26.234 for end-to-end packet switch streaming services. The adoption of HTTP based DASH server into the proposed study enhances the effectiveness of live $8 \mathrm{~K}$ (UHD) video frames streaming and its encoding during a data transmission over a mobile network without affecting its perceptual quality (I.e. PSNR values). The model consider at the HTTP web server unit and client unit communication scenario for uploading and downloading the $8 \mathrm{~K}$ (UHD) video file. The video is uploaded at the server side using the efficient HEVC compression technique. After that, the DASH server is initialized to perform further encoding on the digitalized $8 \mathrm{~K}$ video frames. After successful DASH server initialization and video compression, the server broadcast a request message to the all respective client nodes that are waiting to receive and stream the videos in online buffering. The client establishes a secure TCP/IP integrated connection considering the DASH server's IP address and port number. The successful connection establishment leads to transmitting the $8 \mathrm{~K}$ video frames over a wireless channel. The H.265 compression also takes place during the transmission to encode the video frames. The client receives the video and performs HEVC decoding and watches the video respectively. The compression of bit-stream over a mobile network doesn't affect the video quality regarding perceptual quality and resolution aspects. Moreover, it enhances the perceptual quality to support the $8 \mathrm{~K}$ (UHD) resolutions at the client unit. The next section provides the algorithmic implementation procedures associated with the proposed model.

\section{ALGORITHM IMPLEMENTATION}

This section introduces the analytical modeling and the algorithmic implementation of the proposed HEVC video compression technique. The algorithm implementation of the proposed prototype has been carried out using Matlab tool. The proposed study develops an optimized HEVC based video compression technique, which connects different level of computations to perform HEVC encoding on the real-time video frame streaming without affecting it's perceptual quality. The proposed HEVC protocol aimed to attain maximum retention of $8 \mathrm{~K}$ resolution of transmitted UHD video files. The processing of the video frames attains a different level of optimized computation to ensure an effective and optimal performance trade-off of PSNR and network throughput.

Level-1: Modelling of HEVC for processing input 8K video frames.

Input: $\mathrm{V}_{\mathrm{i}}$ (input video), $\mathrm{f}_{\mathrm{i}}$ (Number of frames), $\mathrm{S}_{1}$ (segment length) where $i \in \mathrm{n}$

Output: $\mathrm{V}_{\mathrm{f}}$ (video frames)

Start

Step 1. Import $\leftarrow \mathrm{V}_{\mathrm{i}}$

Step 2. Initialize $f_{i}, S_{1}$

Step 3. For $\mathrm{i} \leftarrow 1$ : n

Step 4. Apply HEVC Processing-L1

Step 5. $\mathrm{f} \leftarrow$ read pixel information

Step 6. $\quad$ Compute $\leftarrow$ multimedia coefficients

Step 7. $\quad$ End

End 
The above algorithm shows the level-1 computation of HEVC protocol for the efficient improvement of the perceptual quality of a video frame.

Level-2: Modelling of HEVC protocol using H.265 codec

Input: L (length), W (Width), $\mathrm{f}_{\mathrm{n}}$ (frame sequence 1-10)

Output: $\mathrm{C}_{\mathrm{R}}$ (compression Ratio), $\mathrm{C}_{\mathrm{bits}}$ (Number of Compressed bits),

\section{Start:}

Step 1. Initialize $\leftarrow$ f1:f10

Step 2. for $\mathrm{i} \leftarrow 1: \mathrm{n}$

Step 3. $\quad \mathrm{N}_{\mathrm{f}} \leftarrow[\mathrm{f}($ end $)-\mathrm{f}($ start $)+1]$

Step 4. $\quad$ Define $\leftarrow$ MacroblockSize $\left(\mathrm{N}_{\mathrm{f}}\right)$

Step 5. $\mathrm{f} \leftarrow \operatorname{read}(\mathrm{vid}, 1) / /$ read first frame

Step 6. $\quad \mathrm{S}_{\mathrm{f}} \leftarrow$ Get (f)

Step 7. $\mathrm{f} \leftarrow$ imresize (f, [128 128]) //define frame size

Step 8. $\quad I \leftarrow \eta(.2989 * f)+\beta(.5870 * f)+\mu(.1140 * f)$

Step 9. $\quad$ Compute $\leftarrow$ video sequence input

\section{End}

Level-3: Modelling of HEVC protocol using H.265 encoder

Input: L (length), W (Width), $\mathrm{f}_{\mathrm{n}}$ (frame sequence 1-10)

Output: $\mathrm{C}_{\mathrm{R}}$ (compression Ratio), $\mathrm{C}_{\mathrm{bits}}$ (Number of Compressed bits),

\section{Start}

Step 1. Extract $\leftarrow$ input $($ Video_seq)

Step 2. Initialize $\mathrm{f}_{\mathrm{PSNR}}, \mathrm{f}_{\mathrm{BR}}$

Step 3. for $\mathrm{i} \leftarrow 1: \mathrm{n}$

Step 4. VideoSeq_rec $\leftarrow \eta\left(\mathrm{L}, \mathrm{H}, \mathrm{f}_{\mathrm{n}}\right)$

Step 5. $\quad$ Apply $\leftarrow$ HEVC Encoding Level-2

Step 6. $\quad$ Percentage_t $1 \leftarrow(\mathrm{t} 1+\mathrm{t} 2+\mathrm{t} 3 \ldots \mathrm{tn})$

Step 7. $\quad \mathrm{B}_{\text {size }} \leftarrow \mathrm{B}_{\text {size }} * 4$

Step 8. $\quad \mathrm{B}_{\text {stream }} \leftarrow\left[\mathrm{B}_{\text {stream }}{ }^{\prime} 0000\right.$ '] // appending P-frame

Step 9. Compute Comratio $\leftarrow\left[\mathrm{L} * \mathrm{H}^{*}\right.$ Size (VideoSeq_Input, $3)^{*} 8$;
Step 10. Compute $\leftarrow$ PSNR

Step 11. Compute $\leftarrow$ No_original_Bits |

No_CompressedBits

Step 12. End

Step 13. Upload encoded video to the dash server and again perform H.265 standard to enhance the PSNR.

\section{End}

The above-connected algorithms show how different level of optimized HEVC encoding has been performed on a realtime video streaming to increase the perceptual quality of high quality (UHD) video frames. The encoding has also been performed on a real-time video streaming followed by appending $\mathrm{P}$-frame in the header. The primary encoding imposed on the input frames and the processed frame is stored in a temporary matrix. The proposed HEVC method ensures the higher reliability on maintaining pixel information along with the superior reduction of media file size to utilize the network bandwidth effectively. The experimental setup and its respective configuration details are provided below.

- Tool Used: The proposed mechanism is developed, deployed and executed on both 32 bit as well as on 64 bits machine. The development platform was MATLAB.

- Specification: The proposed study has been conducted considering the OFDM wireless channel system OFDM communication and protocol frameworks don't depend on expanded image rates keeping in mind the end goal to accomplish higher information rates. This makes the undertaking of overseeing inter-symbol impedance (ISI) much less complex. OFDM frameworks break the accessible transfer speed into numerous smaller sub-bearers and transmit the information in parallel streams.

\section{RESUlt ANALYSIS}

The proposed system is integrated with HEVC standard to enhance the perceptual quality of video frames for supporting 8 $\mathrm{K}$ (UHD) resolutions. The design and development phase of the proposed model has been carried out using MATLAB 2015 and its two different instances respectively to support the client and server applications. The experimental prototyping also performs a benchmarking by initiating a comparative analysis of the results obtained from the proposed model (H.265) and the conventional H.264 and H.263. The proposed system is found to achieve more precise outcomes (i.e. the enhancement of video quality regarding PSNR) for supporting $8 \mathrm{~K}$ (UHD) resolution based video frames without affecting the cost effectiveness and the complex hardware unit. Different video files (i.e. no of frames) of few seconds are processed and compressed using HEVC encoding to validate the quality of experimental results. 


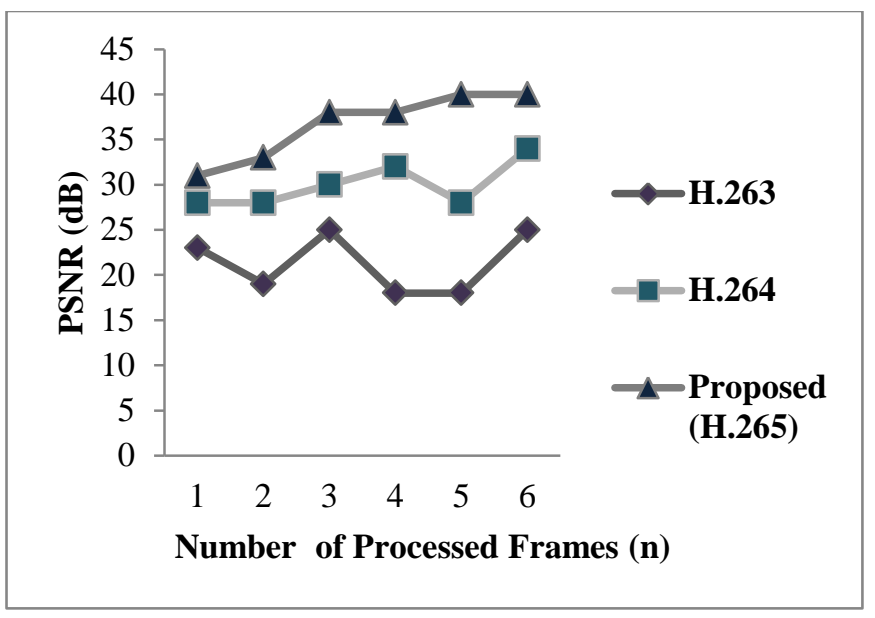

Fig. 2. Perceptual Quality Evaluations

The above-mentioned comparative analysis highlighted in figure 10 depicts that the proposed model not only enhance the perceptual quality of video frames, moreover it also maintains the video compression ratio on standard PSNR (dB) range in between 30-40 (dB). The experimental prototype also suggests that proposed model is capable of maintaining the $8 \mathrm{~K}$ (UHD) video resolutions, which requires very less amount of storage space in DASH servers for both client and server units. It also reduces the computational complexities in comparison with the conventional techniques.

\section{CONCLUSION}

The proposed study performs a more deep investigation on standard definition (SD), High Definition (HD), and Ultra High Definition (UHD). Usually streaming videos over wireless medium will have possible degradation in the video quality even to minor extent; hence, these issues will be addressed in this phase of the study. The study has accomplished a most superior quality of video that supports $8 \mathrm{~K}$ features using H.265. The comparative analysis of the proposed H.265 decoder shows that it effectively enhances the PSNR (perceptual quality of a video signal) by reducing the computational time and space complexities. It also outperforms the conventional H.263 and H.264 standards.

\section{REFERENCES}

[1] I.E. Richardson, "H.264 and MPEG-4 Video Compression: Video Coding for Next-generation Multimedia", John Wiley \& Sons, Science, pp.306, 2004

[2] D. R. Bull, "Communicating Pictures: A Course in Image and Video Coding", Academic Press, Computers, pp. 560, 2014

[3] Y-H Chen, V. Sze, "A Deeply Pipelined CABAC Decoder for HEVC Supporting Level 6.2 High-Tier Applications", IEEE Transactions On Circuits And Systems For Video Technology, Vol. 25, No. 5, 2015

[4] S. Dias, M. Siekmann, S. Bosse, H. Schwarz, "Rate-Distortion Optimized Quantization For HEVC Using Spatial Just Noticeable Distortion", IEEE-European Signal Processing Conference, 2015

[5] T. Nguyen and D. Marpe, "Objective Performance Evaluation of the HEVC Main Still Picture Profile", IEEE Transactions On Circuits And Systems For Video Technology, Vol. 25, No. 5, 2015

[6] S. Panayides, M. S. Pattichis, C. P. Loizou, "An Effective Ultrasound Video Communication System Using Despeckle Filtering and HEVC", IEEE Journal Of Biomedical And Health Informatics, Vol. 19, No. 2, 2015

[7] L. Trzcianowski, "Subjective Assessment for Standard Television Sequences and Videotoms H.264/AVC Video Coding Standard", Journal of Telecommunications and Information technology, 2015

[8] Y. Ye, Y. He, and X. Xiu, "Manipulating Ultra-High Definition Video Traffic", IEEE Computer Society, 2015

[9] G. He, D. Zhou, Y. Li, Z. Chen, "High-Throughput Power-Efficient VLSI Architecture of Fractional Motion Estimation for Ultra-HD HEVC Video Encoding”, IEEE Transactions on Very Large Scale Integration (VLSI) Systems, Vol. 23, No. 12, 2015

[10] Y-J Ahn, T-J Hwang, D-G Sim, and W-J Han, "Implementation of fast HEVC encoder based on SIMD and data-level parallelism", SpringerEURASIP Journal on Image and Video Processing, Vol.16, 2014

[11] S. G. Blasi, I. Zupancic, and E. Izquierdo, "Fast motion estimation discarding low-impact fractional blocks", IEEE-Proceedings of 22nd European Signal Processig Conference, pp.201-215, 2014

[12] T. Diaset, N. Roma and L. Sousa, "Unified transform architecture for AVC, AVS, VC-1 and HEVC high-performance codecs", SpringerEURASIP Journal on Advances in Signal Processing, Vol.108, 2014

[13] F. Mu, L. Song, Z. Luo, X. Wang, X. Yang, "Speed Up HEVC Encoder by Precoding with H.264", IEEE-Annual Summit and Conference AsiaPacific Signal and Information Processing Association, pp.1-6, 2014 